\title{
Fatal hemorrhagic pneumonia in patients with hematologic diseases and Stenotrophomonas maltophilia bacteremia: a retrospective study
}

\section{Lixia ZHU}

Zhejiang University First Affiliated Hospital Department of Hematology

\section{Lulu Wang}

Zhejiang University First Affiliated Hospital Department of Hematology

\section{Yuping Zhang}

Zhejiang University School of Medicine First Affiliated Hospital

\section{Rongrong Chen}

Zhejiang University First Affiliated Hospital Department of Hematology

\section{Xueying Li}

Zhejiang University First Affiliated Hospital Department of Hematology

Jianai Sun

Zhejiang University First Affiliated Hospital Department of Hematology

\section{De Zhou}

Zhejiang University First Affiliated Hospital Department of Hematology

\section{Mingyu Zhu}

Zhejiang University First Affiliated Hospital Department of Hematology

\section{Xiaolong Zheng}

Zhejiang University First Affiliated Hospital Department of Hematology

\section{Li Li}

Zhejiang University First Affiliated Hospital Department of Hematology Jingjing Zhu

Zhejiang University First Affiliated Hospital Department of Hematology

\section{Mixue Xie}

Zhejiang University First Affiliated Hospital Department of Hematology

\section{Xiudi Yang}

Zhejiang University First Affiliated Hospital Department of Hematology

\section{Wenjuan Yu}

Zhejiang University First Affiliated Hospital Department of Hematology

\section{Hongyan Tong}

Zhejiang University First Affiliated Hospital Department of Hematology

Honghu Zhu 
Zhejiang University First Affiliated Hospital Department of Hematology

\section{Wanzhuo Xie}

Zhejiang University First Affiliated Hospital Department of Hematology

\section{Jie Jin}

Zhejiang University First Affiliated Hospital Department of Hematology

\section{YeXiujin ( $\sim$ yxjsunny@zju.edu.cn )}

Zhejiang University First Affiliated Hospital Department of Hematology https://orcid.org/0000-00024553-2751

\section{Research article}

Keywords: Stenotrophomonas maltophilia, Bacteremia, Hematologic diseases, Hemorrhagic pneumonia, mortality

Posted Date: March 31st, 2021

DOI: https://doi.org/10.21203/rs.3.rs-366323/v1

License: (a) (1) This work is licensed under a Creative Commons Attribution 4.0 International License. Read Full License

Version of Record: A version of this preprint was published at BMC Infectious Diseases on July 31st, 2021. See the published version at https://doi.org/10.1186/s12879-021-06420-0. 


\section{Abstract \\ Background}

Fatal hemorrhagic pneumonia is one of the most severe manifestations of Stenotrophomonas maltophilia (SM) infections. Here, we aimed to investigate the clinical characteristics of SM bacteremia and to identify the risk factors of hemorrhagic pneumonia caused by SM in patients with hematologic diseases.

\section{Methods}

The clinical records of 55 patients diagnosed with hematologic diseases and SM bacteremia were retrospectively reviewed. We compared patients' clinical characteristics and outcomes between the hemorrhagic pneumonia group and non-hemorrhagic pneumonia group.

\section{Results}

Twenty-seven (49.1\%) patients developed hemorrhagic pneumonia. The overall mortality rate of SM bacteremia was $67.3 \%$. The 30 -day mortality rate was significantly higher in patients with hemorrhagic pneumonia than those without hemorrhagic pneumonia $(85.2 \%$ and $50.0 \%$, respectively, $P=0.003)$. Compared with the non-hemorrhagic pneumonia group, patients in the hemorrhagic pneumonia group were older and showed clinical manifestations as higher proportions of isolated SM in sputum culture, neutropenia and elevated procalcitonin. Multivariate analysis showed that neutropenia, high levels of procalcitonin, prior tigecycline therapy within 1 month were independent risk factors associated with hemorrhagic pneumonia.

\section{Conclusions}

Hematologic patients with SM bacteremia who have neutropenia, high level of procalcitonin and prior tigecycline therapy within 1 month should be early treated with combined effective antibiotics and best supportive interventions to avoid life-threatening hemorrhagic pneumonia.

\section{Introduction}

Stenotrophomonas maltophilia (SM) is a non-fermentative gram-negative bacterium without highly virulent widely found in nature, and rarely causes infection in the normal immune population [1]. However, SM has been becoming a common cause of opportunistic infections in immunocompromised patients, especially in patients diagnosed with hematologic diseases, and can cause serious infections, such as bacteremia or hemorrhagic pneumonia [2-6]. 
Due to the intrinsic resistance of SM to many broad-spectrum antimicrobial agents, it is difficult to determine the appropriate antibiotic treatment before the SM is detected $[2,7]$. Therefore, previous studies have shown a very poor prognosis in the cases of SM bacteremia, and the overall mortality was ranged from 35 to 75\% [8-12]. Noteworthy, in patients with hematologic malignancies and/or hematopoietic stem cell transplantation (HSCT) recipients, SM can cause fatal hemorrhagic pneumonia, and the mortality is nearly $100 \%[10,12-17]$. At present, only a few reports have performed a statistical analysis to identify the risk factors of hemorrhagic pneumonia caused by SM $[10,12]$.

In this study, we identified cases of SM bacteremia in patients with hematologic disorders and performed a retrospective review of these patients' clinical characteristics and outcomes. Further, we attempted to confirm the risk factors of SM bacteremia with hemorrhagic pneumonia.

\section{Materials And Methods}

\section{Patients}

This study was conducted at a 318-bed hematology department, the first affiliated hospital, College of Medicine, Zhejiang University, in China. From January 2015 to July 2019, all patients who were diagnosed with hematologic diseases and had positive blood cultures for SM were included in the study, with each patient being included only once at the time of initial blood culture. All patients were categorized into 2 groups: hemorrhagic pneumonia group and non-hemorrhagic pneumonia group. Clinical and microbiological characteristics were compared between the two groups to identify the risk factors for hemorrhagic pneumonia in SM bacteremia patients.

\section{Data collection}

We retrospectively reviewed the electronic medical records for all enrolled patients and extracted the clinical data. The data included the following: age, gender, underlying hematologic disease, therapeutic treatment for hematologic diseases, sputum culture, polymicrobial bacteremia, laboratory tests, antibiotics susceptibility testing results, prior antibiotic therapy within 1 month, indwelling central venous catheter, inadequate initial empirical antimicrobial treatment and mortality.

\section{Definitions}

SM bacteremia was defined as one or more positive blood cultures with clinical symptoms of infection. SM bacteremia with hemorrhagic pneumonia was diagnosed as follows:1) SM isolated from a blood culture, with or without SM in sputum culture at the same time, 2) newly detected imaging findings by chest X-ray or computed tomography (CT) scan when the blood culture was positive, 3) with pulmonary hemorrhage symptoms, such as continuous blood in sputum or hemoptysis. Polymicrobial bacteremia was defined as the isolation of another pathogen within $24 \mathrm{~h}$ of index SM isolate, satisfying CDC criteria for BSI [18]. Neutropenia was defined as an absolute neutrophil count of $<500 / u L$ at the onset of bacteremia. Inadequate initial antimicrobial treatment was defined as no administration of at least one 
agent which showed sensitive in vitro antibiotic susceptibility test before obtaining the bacteria cultivation results.

\section{Blood culture and drug sensitivity test}

The BacT/Alert 3D (bioMérieux, France) automated blood culture system was used. Species identification was performed by the automated Vitek 2 system with the matched gram-negative (GN) test card (bioMérieux, France). Antimicrobial susceptibility was tested with the agar dilution method. The antibiotics tested included TMP-SMX (trimethoprim-sulfamethoxazole), cefoperazone/sulbactam, minocycline, levofloxacin and tigecycline. The results were interpreted according to the 2018 Clinical and Laboratory Standards Institute (CLSI) criteria[19].

\section{Statistical analysis}

All statistical analyses were performed using SPSS version 22.0 for Windows. The independent sample ttest was used for continuous variables and the Chi-square test or Fisher's exact test was used for categorical variables. The survival curves were performed by the Kaplan-Meier method. Univariate and multivariable logistic regression was used to evaluate the risk factors of hemorrhagic pneumonia in hematologic patients with SM bacteremia. Variables with $P$-value of less than 0.10 in univariate analyses were included in a multivariable logistic regression analysis. Results from the univariate and multivariate analysis are expressed as an odds ratio (OR) and 95\% confidence interval (Cl). Statistical tests were twosided, and significance was defined as $P$-value $<0.05$.

\section{Results}

\section{Study population}

Of the 288 patients with SM positive culture in the hematology department during the study period, 55 (19.1\%) with SM bacteremia were included (Figure 1). The median age was 51 (range 12-72) years old and $56.4 \%$ were male in our study population. The clinical characteristics of this cohort were shown in Table 1. The most common underlying diseases diagnosed when admitted to hospital were acute myeloid leukemia (AML) (31 cases, $56.4 \%$ ), followed by acute lymphoblastic leukemia (ALL) (12 cases, $21.8 \%$ ), non-Hodgkin's lymphoma (NHL) $₫ 6$ cases, $10.6 \%$, aplastic anemia (AA) (3 cases, $5.5 \%$ ), hemophagocytic syndrome (HLH) (2 cases, 3.6\%) and myelodysplastic syndrome (MDS) $₫ 1$ case, $1.8 \% \rrbracket$. Thirteen patients $(23.6 \%)$ received HSCT. In addition to SM, 11 patients $(20 \%)$ were also concomitant with other microbial bacteremia, included Klebsiella pneumonia in 3 cases, Escherichia coli in 3 cases, Staphylococcus haemolyticus in 2 cases, Pseudomonas cepacia in 2 cases, Staphylococcus epidermidis in 1 case, and Enterococcus faecium in 1 case. Fifty-two (94.5\%) patients with SM bacteremia had thrombocytopenia and $50(90 \%)$ patients' platelet counts were less than 50,000/uL. Bleeding was one of the most common clinical manifestations in this study. There were $30(54.5 \%)$ patients with hemoptysis, $11(20.0 \%)$ patients with gastrointestinal bleeding and $5(9.1 \%)$ patients with intracranial hemorrhage. 
Hemorrhagic pneumonia was confirmed in 27(49.1\%) patients. However, SM was only identified in 14 (25.5\%) patients from sputum culture simultaneously.

The cohort was categorized into hemorrhagic pneumonia group and non-hemorrhagic pneumonia group (27 cases and 28 cases, respectively). The comparisons of clinical characteristics between the two groups were listed in Table 1. The median age was 56 years old in the hemorrhagic pneumonia group, which was older than the 48.5 years observed in the non-hemorrhagic pneumonia group $(P=0.049)$. There were more patients $(40.7 \%)$ isolated SM from sputum in the hemorrhagic pneumonia group. The proportions of neutropenia and elevated procalcitonin were significantly higher in the hemorrhagic pneumonia group than the non-hemorrhagic pneumonia group ( $P=0.016$ and $P=0.003$, respectively). There were no significant differences for patients' gender, polymicrobial bacteremia, anemia, platelet count, coagulation function, underlying hematologic diseases, C-reactive protein level, inadequate initial antimicrobial treatment and prior courses of chemotherapy between the two groups.

\section{Mortality}

The overall mortality was $37(67.3 \%)$, and all patients died within 30 days after SM bacteremia onset. It was noteworthy that $20(36.4 \%)$ patients died before obtained the positive blood culture reports, and all these patients had hemorrhagic pneumonia. The 30-day mortality rate was significantly higher in patients with hemorrhagic pneumonia than those without hemorrhagic pneumonia ( $85.2 \%$ and $50.0 \%$, respectively, $P=0.003)$, showed in Figure 2a. In this study, 35 (63.6\%) patients received inadequate initial antimicrobial treatment. The 30 -day mortality rate was $77.1 \%$ in patients who received inadequate initial antimicrobial treatment, which was significantly higher than that in patients treated with adequate initial antimicrobial $(50.0 \%, P=0.040)$, showed in Figure $2 b$.

\section{Previous antimicrobial therapy within 1 month before BSI onset}

All patients in this study were administrated with antibiotics before SM bacteremia onset, either due to a previous other infection or as an empirical therapy for unknown origin infection (Table 2). The majority of patients received carbapenems ( 46 cases, $83.6 \%$ ) and/or antifungal drugs ( 45 cases, $81.8 \%$ ). But there were only a few patients who had treated with TMP-SMX ( 3 cases, $5.5 \%$ ). It was to be observed that a total of $23(41.8 \%)$ cases received tigecycline, and tigecycline was more frequently prescribed in the hemorrhagic pneumonia group ( $59.3 \%$ and $25 \%$, respectively, $P=0.010)$.

\section{In vitro antimicrobial susceptibility}

The antimicrobial susceptibilities of SM isolate in vitro are shown in Figure 3. All patients were tested for TMP-SMX sensitivity, with a sensitivity rate of $89.09 \%$. Susceptibility to levofloxacin, minocycline, cefoperazone/sulbactam was $83.3 \%$ (40/48), 100\% (46/46) and 77.8\% (35/45), respectively. There was no significant difference in susceptibility to four antibiotics mentioned above between the hemorrhagic pneumonia group and the non-hemorrhagic pneumonia group. In the present study, only six cases performed susceptibility tests to tigecycline, and the susceptibility is $83.3 \%(5 / 6)$. 


\section{Risk factors associated with hemorrhagic pneumonia}

For patients with SM bacteremia, once hemorrhagic pneumonia develops, the patients' conditions always rapidly deteriorate and nearly all these patients die in a very short time. Univariate analysis showed that neutropenia, high levels of procalcitonin $(>0.5 \mathrm{ug} / \mathrm{L})$ and prior tigecycline therapy within 1 month were significant risk factors for hemorrhagic pneumonia in SM bacteremia patients. In multivariate analysis showed in Table 3, independent risk factors associated with hemorrhagic pneumonia were neutropenia $(\mathrm{HR}=4.965,95 \% \mathrm{Cl}[1.010 ; 24.403)], P=0.049)$, high levels of procalcitonin $(\mathrm{HR}=11.241,95 \% \mathrm{Cl}[1.825$; 69.241], $P=0.009)$, prior tigecycline therapy within 1 month $(\mathrm{HR}=4.465,95 \% \mathrm{Cl}[1.108 ; 18.000], P=0.035)$.

\section{Discussion}

In the present study, the overall mortality rate of SM bacteremia in patients with hematologic diseases was $67.3 \%$, which was comparable to that obtained in past studies, ranging from $35-75 \%$ [8-12]. The mortality rate of SM bacteremia with hemorrhagic pneumonia was significantly higher than those without hemorrhagic pneumonia. Neutropenia, high level of procalcitonin and prior tigecycline therapy were found to be independent risk factors associated with hemorrhagic pneumonia in hematologic patients with SM bacteremia.

Hemorrhagic pneumonia is one of the most severe SM infections. The exact mechanism by which SM causes hemorrhage is still unclear. A study by Windhorst et al. [20], suggests that StmPr1, a protease of the subtilase family secreted by SM, can degrade the collagen and fibroblasts in vitro. This process might lead to tissue invasion, destruction of the alveolar micro vessels and hemorrhage. This type of infection is usually characterized as a rapidly progressive and fatal disease course in patients with hematologic malignancy. Almost all patients die within a few days after the onset of hemoptysis and before the isolate is detected from blood or sputum culture. In our study, 23 of the 27 (85.2\%) SM bacteremia patients with hemorrhagic pneumonia died within the hospitalization period. In this group of dead patients, 20 patients died within one week after the onset of hemoptysis, and 13 cases obtained the identified SM isolation after death. There were 4 patients with successful treatment outcomes after early combination therapy with TMP/SMX, cefoperazone/sulbactam, moxifloxacin, and/or tigecycline, and rapid recovery of neutropenia. There are only two patients who were diagnosed with hematologic malignancies and hemorrhagic pneumonia due to SM infection and successfully treated after early combined therapy with TMP/SMX, polymyxin, and/or moxifloxacin were reported in the previous study [21].

Previous literature has proven that inadequate initial antimicrobial treatment is an independent adverse prognostic factor for SM bacteremia[22]. In the present study, the mortality rate is $77.1 \%$ in the patients who received inadequate initial treatment for $\mathrm{SM}$, which is significantly higher than that in the patients treated with adequate initial antimicrobial (50.0\%). The rate of receiving the adequate initial antimicrobial treatment was similar between the SM bacteremia with hemorrhagic pneumonia group and the SM bacteremia without hemorrhagic pneumonia group. However, the mortality rate in the SM bacteremia with 
hemorrhagic pneumonia group was significantly higher. This indicates that hemorrhagic pneumonia caused by SM is a severe condition and difficult to save a patient once it develops. Therefore, it is important to clarify risk factors for hemorrhagic pneumonia caused by SM, and then to promote early diagnosis and treatment of SM bacteremia for preventing the progression to hemorrhagic pneumonia.

A previous review summarized the clinical information of 30 cases of hemorrhagic pneumonia caused by SM [10]. The authors found that severe neutropenia ( $<100 \mathrm{uL}$ ) was the most important risk factor for hemorrhagic pneumonia induced by SM. In the present study as well, 24 of the 27(88.9\%) SM bacteremia patients with hemorrhagic pneumonia were neutropenic $(<500 \mathrm{uL})$. This maybe explain why SM bacteremia is likely to occur in immunocompromised patients, and why hemorrhagic pneumonia induced by SM is always developed in patients with hematologic malignancy. Neutropenia due to the hematologic disorder itself or treatment for the hematologic disorder may be an important factor for susceptibility to SM bacteremia and progression to hemorrhagic pneumonia.

Procalcitonin is a prohormone of calcitonin, which is highly upregulated during the acute phase of sepsis. Procalcitonin, which has a short half-life, is valuable and sensitive in predicting bacteremia and distinguishing Gram-negative sepsis in hematologic patients with febrile neutropenia [23-27]. A metaanalysis review showed that an elevated procalcitonin level was associated with an increased risk of mortality in pneumonia patients, especially for critically ill patients [28]. Our study found a high level of procalcitonin was another risk factor for developing hemorrhagic pneumonia in SM bacteremia patients.

Tigecycline is a potential alternative antimicrobial for SM infection, and the susceptibility to tigecycline is ranged from 70.6-90.6\% according to previous studies [29-31]. However, our study showed that prior tigecycline therapy was an independent risk factor for developing hemorrhagic pneumonia caused by SM, and empirical antimicrobial treatment with tigecycline cannot reduce the mortality rate (63.6\%). The reason may be due to the side effects of tigecycline. It was previously reported that the use of tigecycline might induce coagulopathy usually manifested as prolongation of prothrombin time (PT) and activated partial thromboplastin time (APTT) and a reduction in the fibrinogen level in a dose-dependent manner[32]. The most severe adverse event is bleeding. This view was confirmed by our study again, which showed a higher incidence of prolonged APTT in patients received prior tigecycline therapy (87.0\% vs $\left.56.3 \%, P=0.015, \chi^{2}=5.908\right)$. On the other hand, it was identified that the resistance to tigecycline was easily selected during exposure to this antimicrobial and cross-resistance to other antimicrobials, such as aztreonam and quinolones, was also presented [33]. Unfortunately, in our study, there were only six cases finished susceptibility test to tigecycline in vitro, and only one strain showed resistance. The rate of resistance to trimethoprim-sulfamethoxazole (TMP-SMZ) was higher in the prior tigecycline therapy group $(17.4 \%$ vs $6.3 \%)$, which was no significantly statistical difference $(P=0.223)$.

The study still has several limitations. First of all, $59.3 \%$ of cases did not obtain a positive result of SM in sputum culture in the hemorrhagic pneumonia group. It might lead to the argument about whether SM was the true pathogen of hemorrhagic pneumonia. For this special group of patients, sputum culture was difficult to carry out under certain conditions, such as the sudden appearance of massive hemoptysis. 
Besides, the majority of these patients had thrombocytopenia, so it was unable to perform bronchoalveolar lavage fluid culture to improve the positive rate of culture. According to our definition, the blood cultures of all patients were positive for SM. Then combined with clinical and imaging findings, we reasonably identified that hemorrhagic pneumonia was caused by SM. On the other hand, the antimicrobial susceptibilities of SM isolates were insufficient. There were only a few cases completed the susceptibility test to tigecycline. Consequently, it is difficult to confirm the relationship between the susceptibility to tigecycline and the development of hemorrhagic pneumonia.

In conclusion, our study suggested that SM bacteremia with hemorrhagic pneumonia developed in patients with hematologic diseases was a condition with rapid progression and high mortality. Besides, hematologic patients with SM bacteremia who have neutropenia, high level of procalcitonin and prior tigecycline therapy within 1 month should be early treated with combined effective antibiotics and best supportive interventions to avoid life-threatening hemorrhagic pneumonia.

\section{Declarations}

Ethical approval This study was approved by the clinical research ethics committee of the first affiliated hospital, college of medicine, Zhejiang University.

Conflict of interest The authors declare that they have no conflict of interest.

Funding The research was supported by a grant from Medical Health Science and Technology Project of Zhejiang Provincial Health Commission [No.2017KY059].

Authors' contributions Lixia Zhu and Lulu Wang conducted the hospital chart review, interpreted data, drafted the work and wrote the final report. Yuping Zhang contributed the interpretation of microbiologic data. Rongrong Chen, Xueying Li, Jianai Sun, De Zhou, Mingyu Zhu, Xiaolong Zheng, Li Li, Jingjing Zhu, Mixue Xie, Xiudi Yang conducted the hospital chart review and contributed the data interpretation. Wenjuan Yu, Hongyan Tong, Honghu Zhu, Wanzhuo Xie and Jie Jin participated in critical revision of manuscript. Xiujin Ye conceptualized the study and contributed to data interpretation. All authors read and approved the final manuscript.

Acknowledgments The authors would like to express their sincere thanks to the patients for their contributions to this study.

\section{References}

1. Looney WJ, Narita M, Mühlemann K. Stenotrophomonas maltophilia: an emerging opportunist human pathogen. The Lancet Infectious diseases. 2009;9(5):312-23.

2. Micozzi A, Venditti M, Monaco M, Friedrich A, Taglietti F, Santilli S, et al. Bacteremia due to Stenotrophomonas maltophilia in patients with hematologic malignancies. Clinical infectious diseases : an official publication of the Infectious Diseases Society of America. 2000;31(3):705-11. 
3. Al-Anazi KA, Al-Jasser AM. Infections Caused by Stenotrophomonas maltophilia in Recipients of Hematopoietic Stem Cell Transplantation. Frontiers in oncology. 2014;4:232.

4. Cho SY, Lee DG, Choi SM, Park C, Chun HS, Park YJ, et al. Stenotrophomonas maltophilia bloodstream infection in patients with hematologic malignancies: a retrospective study and in vitro activities of antimicrobial combinations. BMC infectious diseases. 2015;15:69.

5. Baumrin E, Piette EW, Micheletti RG. Stenotrophomonas maltophilia: an emerging multidrug-resistant opportunistic pathogen in the immunocompromised host. BMJ case reports. 2017;2017.

6. Guerci P, Bellut H, Mokhtari M, Gaudefroy J, Mongardon N, Charpentier C, et al. Outcomes of Stenotrophomonas maltophilia hospital-acquired pneumonia in intensive care unit: a nationwide retrospective study. Critical care (London, England). 2019;23(1):371.

7. Metan G, Uzun O. Impact of initial antimicrobial therapy in patients with bloodstream infections caused by Stenotrophomonas maltophilia. Antimicrobial agents and chemotherapy. 2005;49(9):3980-1.

8. Sumida K, Chong Y, Miyake N, Akahoshi T, Yasuda M, Shimono N, et al. Risk Factors Associated with Stenotrophomonas maltophilia Bacteremia: A Matched Case-Control Study. PloS one. 2015;10(7):e0133731.

9. Kim SH, Cho SY, Kang Cl, Seok H, Huh K, Ha YE, et al. Clinical predictors of Stenotrophomonas maltophilia bacteremia in adult patients with hematologic malignancy. Annals of hematology. 2018;97(2):343-50.

10. Mori M, Tsunemine H, Imada K, Ito K, Kodaka T, Takahashi T. Life-threatening hemorrhagic pneumonia caused by Stenotrophomonas maltophilia in the treatment of hematologic diseases. Annals of hematology. 2014;93(6):901-11.

11. Kim EJ, Kim YC, Ahn JY, Jeong SJ, Ku NS, Choi JY, et al. Risk factors for mortality in patients with Stenotrophomonas maltophilia bacteremia and clinical impact of quinolone-resistant strains. BMC infectious diseases. 2019;19(1):754.

12. Imoto W, Yamada K, Yamairi K, Shibata W, Namikawa H, Yukawa S, et al. Clinical Characteristics of Rapidly Progressive Fatal Hemorrhagic Pneumonia Caused by Stenotrophomonas maltophilia. Internal medicine (Tokyo, Japan). 2020;59(2):193-8.

13. Araoka H, Fujii T, Izutsu K, Kimura M, Nishida A, Ishiwata K, et al. Rapidly progressive fatal hemorrhagic pneumonia caused by Stenotrophomonas maltophilia in hematologic malignancy. Transplant infectious disease : an official journal of the Transplantation Society. 2012;14(4):355-63.

14. Tada K, Kurosawa S, Hiramoto N, Okinaka K, Ueno N, Asakura Y, et al. Stenotrophomonas maltophilia infection in hematopoietic SCT recipients: high mortality due to pulmonary hemorrhage. Bone marrow transplantation. 2013;48(1):74-9.

15. Gutierrez C, Pravinkumar E, Balachandran D, Schneider V. Fatal hemorrhagic pneumonia: Don't forget Stenotrophomonas maltophilia. Respiratory medicine case reports. 2016;19:12-4.

16. Katayama O, Kitagawa T, Onaka T, Yonezawa A. Rapidly progressive hemorrhagic pneumonia caused by Stenotrophomonas maltophilia occurring early after bone marrow transplantation in a patient 
with myelodysplastic syndrome. Rinsho ketsueki. 2017;58(4):323-5.

17. Kim SH, Cha MK, Kang Cl, Ko JH, Huh K, Cho SY, et al. Pathogenic significance of hemorrhagic pneumonia in hematologic malignancy patients with Stenotrophomonas maltophilia bacteremia: clinical and microbiological analysis. European journal of clinical microbiology \& infectious diseases : official publication of the European Society of Clinical Microbiology. 2019;38(2):285-95.

18. Horan TC, Andrus M, Dudeck MA. CDC/NHSN surveillance definition of health care-associated infection and criteria for specific types of infections in the acute care setting. American journal of infection control. 2008;36(5):309-32.

19. Humphries RM, Ambler J, Mitchell SL, Castanheira M, Dingle T, Hindler JA, et al. CLSI Methods Development and Standardization Working Group Best Practices for Evaluation of Antimicrobial Susceptibility Tests. Journal of clinical microbiology 2018;56(4).

20. Windhorst S, Frank E, Georgieva DN, Genov N, Buck F, Borowski P, et al. The major extracellular protease of the nosocomial pathogen Stenotrophomonas maltophilia: characterization of the protein and molecular cloning of the gene. The Journal of biological chemistry. 2002;277(13):11042-9.

21. Penagos SC, Giraldo N, Vallejo C, McEwen O, Hidron A. First report of survival in two patients with hematologic malignancy and Stenotrophomonas maltophilia hemorrhagic pneumonia treated with trimethoprim-sulfamethoxazole-based combination antibiotic therapy. Journal of infection and chemotherapy : official journal of the Japan Society of Chemotherapy. 2020;26(4):397-9.

22. Bao H, Qiao Y, Liu D, Chen J, Wu X, Hu X, et al. The clinical impact of Stenotrophomonas maltophilia bacteremia on the 30-day mortality rate in patients with hematologic disorders: a single-institution experience. Infection. 2020;48(2):205-12.

23. Koivula I, Hämäläinen S, Jantunen E, Pulkki K, Kuittinen T, Nousiainen T, et al. Elevated procalcitonin predicts Gram-negative sepsis in haematological patients with febrile neutropenia. Scandinavian journal of infectious diseases. 2011;43(6-7):471-8.

24. von Lilienfeld-Toal M, Dietrich MP, Glasmacher A, Lehmann L, Breig P, Hahn C, et al. Markers of bacteremia in febrile neutropenic patients with hematological malignancies: procalcitonin and IL-6 are more reliable than C-reactive protein. European journal of clinical microbiology \& infectious diseases : official publication of the European Society of Clinical Microbiology. 2004;23(7):539-44.

25. Juutilainen A, Hämäläinen S, Pulkki K, Kuittinen T, Nousiainen T, Jantunen E, et al. Biomarkers for bacteremia and severe sepsis in hematological patients with neutropenic fever: multivariate logistic regression analysis and factor analysis. Leukemia \& lymphoma. 2011;52(12):2349-55.

26. Yang M, Choi SJ, Lee J, Lee DG, Kim YJ, Park YJ, et al. Serum procalcitonin as an independent diagnostic markers of bacteremia in febrile patients with hematologic malignancies. PloS one. 2019;14(12):e0225765.

27. Yunus I, Fasih A, Wang Y. The use of procalcitonin in the determination of severity of sepsis, patient outcomes and infection characteristics. PloS one. 2018;13(11):e0206527.

28. Liu D, Su LX, Guan W, Xiao K, Xie LX. Prognostic value of procalcitonin in pneumonia: A systematic review and meta-analysis. Respirology. 2016;21(2):280-8. 
29. Tekçe YT, Erbay A, Cabadak H, Sen S. Tigecycline as a therapeutic option in Stenotrophomonas maltophilia infections. Journal of chemotherapy. 2012;24(3):150-4.

30. Pfaller MA, Huband MD, Streit JM, Flamm RK, Sader HS. Surveillance of tigecycline activity tested against clinical isolates from a global (North America, Europe, Latin America and Asia-Pacific) collection (2016). International journal of antimicrobial agents. 2018;51(6):848-53.

31. Zhao J, Liu Y, Liu Y, Wang D, Ni W, Wang R, et al. Frequency and Genetic Determinants of Tigecycline Resistance in Clinically Isolated Stenotrophomonas maltophilia in Beijing, China. Frontiers in microbiology. 2018;9:549.

32. Cui N, Cai H, Li Z, Lu Y, Wang G, Lu A. Tigecycline-induced coagulopathy: a literature review. International journal of clinical pharmacy. 2019;41(6):1408-13.

33. Blanco P, Corona F, Martinez JL. Mechanisms and phenotypic consequences of acquisition of tigecycline resistance by Stenotrophomonas maltophilia. The Journal of antimicrobial chemotherapy. 2019;74(11):3221-30.

\section{Tables}

Table 1. Demographic and clinical characteristics of hematologic patients with SM bacteremia 


\begin{tabular}{|c|c|c|c|c|}
\hline Characteristics & $\begin{array}{l}\text { Patients } \\
(\mathrm{N}=55)\end{array}$ & $\begin{array}{l}\text { Hemorrhagic } \\
\text { pneumonia } \\
(n=27)\end{array}$ & $\begin{array}{l}\text { Non-hemorrhagic } \\
\text { pneumonia } \\
(n=28)\end{array}$ & $\begin{array}{l}P \text { - } \\
\text { value* }\end{array}$ \\
\hline Gender, male, No. (\%) & $31(56.4)$ & $17(63.0)$ & $14(50.0)$ & 0.333 \\
\hline Age, years, median(range) & $51(12-72)$ & $56(17-72)$ & $48.5(12-71)$ & 0.049 \\
\hline $\begin{array}{l}\text { Positive for SM in sputum culture, } \\
\text { No. (\%) }\end{array}$ & $14(25.5)$ & 11(40.7) & $3(10.7)$ & 0.011 \\
\hline Polymicrobial bacteremia, No. (\%) & $11(20.0)$ & $4(14.8)$ & $7(25.0)$ & 0.345 \\
\hline Neutropenia, No. (\%) & $41(74.5)$ & $24(88.9)$ & $17(60.7)$ & 0.016 \\
\hline Hemoglobin $<60 \mathrm{~g} / \mathrm{L}$, No. (\%) & $31(56.4)$ & $16(59.3)$ & $15(53.6)$ & 0.671 \\
\hline Platelet $<50,000 /$ uL, No. (\%) & $50(90.9)$ & $25(92.6)$ & 25(89.3) & 1.000 \\
\hline Fibrinogen $<2 g / L$, No. $(\%)$ & $11(20.0)$ & $7(25.9)$ & $4(14.3)$ & 0.281 \\
\hline PT>13.5s, No. (\%) & $22(40.0)$ & 14(51.9) & $8(28.6)$ & 0.078 \\
\hline APTT>33.5s, No. (\%) & $38(69.1)$ & $21(77.8)$ & $17(60.7)$ & 0.171 \\
\hline D-Dimer>700ug/L FEU, No. (\%) & $45(81.8)$ & 23(85.2) & $22(78.6)$ & 0.775 \\
\hline Albumin<30g/L, No. (\%) & $22(40.0)$ & $12(44.4)$ & $10(35.7)$ & 0.509 \\
\hline $\begin{array}{l}\text { C-reactive protein } \geq 10 \mathrm{mg} / \mathrm{dl} \text {, No. } \\
(\%)\end{array}$ & $31(56.4)$ & $17(68.0)$ & 14(51.9) & 0.236 \\
\hline Procalcitonin $>0.5 \mathrm{ug} / \mathrm{L}$, No. (\%) & $41(74.5)$ & $25(92.6)$ & $16(57.1)$ & 0.003 \\
\hline Hematological diseases & & & & 0.544 \\
\hline AML, No. (\%) & $31(56.4)$ & 15(55.6) & $16(57.1)$ & \\
\hline ALL, No. (\%) & $12(21.8)$ & $7(25.9)$ & $5(17.9)$ & \\
\hline NHL, No. (\%) & $6(10.9)$ & $3(11.1)$ & $3(10.7)$ & \\
\hline MDS, No. (\%) & $1(1.8)$ & $1(3.7)$ & $0(0.0)$ & \\
\hline AA, No. (\%) & $3(5.5)$ & $0(0.0)$ & $3(10.7)$ & \\
\hline HLH, No. (\%) & $2(3.6)$ & $1(3.7)$ & 1(3.6) & \\
\hline Chemotherapy in 30-day, No. (\%) & $43(78.2)$ & $21(77.8)$ & $22(78.6)$ & 0.943 \\
\hline $\begin{array}{l}\text { Inadequate initial antimicrobial } \\
\text { treatment, No. }(\%)\end{array}$ & $35(63.6)$ & $16(59.3)$ & $19(67.9)$ & 0.508 \\
\hline Central venous catheter, No. (\%) & $48(87.3)$ & $25(92.6)$ & 23(82.1) & 0.449 \\
\hline HSCT, No. (\%) & $13(23.6)$ & $6(22.2)$ & $7(25.0)$ & 0.808 \\
\hline
\end{tabular}




\begin{tabular}{|lllll|}
\hline 0 & $18(32.7)$ & $7(25.9)$ & $11(39.3)$ & \\
\hline$\geq 1$ & $37(67.3)$ & $20(74.1)$ & $17(60.7)$ & \\
\hline Chemotherapy in 30-day, No. (\%) & $43(78.2)$ & $21(77.8)$ & $22(78.6)$ & 0.943 \\
\hline 30-day mortality, No. (\%) & $37(67.3)$ & $23(85.2)$ & $14(50.0)$ & 0.003 \\
\hline
\end{tabular}

Abbreviations: PT, prothrombin time; APTT, activated partial thromboplastin time; AML, acute myeloid leukemia; ALL, acute lymphoblastic leukemia; NHL, non-Hodgkin's lymphoma; MDS, myelodysplastic syndrome; $A A$, aplastic anemia; $\mathrm{HLH}$, hemophagocytic syndrome; $\mathrm{HSCT}$, hematopoietic stem cell transplantation.

*Significant difference between hemorrhagic pneumonia group and non-hemorrhagic pneumonia group

\section{Table 2. Previous antimicrobial therapy within 1 month before SM bacteremia onset}

\begin{tabular}{|llllr|}
\hline Antibiotics & $\begin{array}{l}\text { Total } \\
(\mathrm{N}=55)\end{array}$ & $\begin{array}{l}\text { Hemorrhagic } \\
\text { pneumonia(N=27) }\end{array}$ & $\begin{array}{l}\text { Non-hemorrhagic } \\
\text { pneumonia (N=28) }\end{array}$ & $\begin{array}{c}\text { P- } \\
\text { value }\end{array}$ \\
\hline Carbapenems, No. (\%) & $46(83.6)$ & $23(85.2)$ & $23(82.1)$ & 1.000 \\
\hline${ }^{a}$ Anti-MRSAs, No. (\%) & $38(69.1)$ & $16(59.3)$ & $22(78.6)$ & 0.121 \\
\hline Aminoglycoside, No. (\%) & $5(9.1)$ & $3(11.1)$ & $2(7.1)$ & 0.966 \\
\hline Tigecycline, No. (\%) & $23(41.8)$ & $16(59.3)$ & $7(25.0)$ & 0.010 \\
\hline Fluoroquinolones, No. (\%) & $11(20.0)$ & $6(22.2)$ & $5(17.9)$ & 0.686 \\
\hline TMP-SMX, No. (\%) & $3(5.5)$ & $0(0.0)$ & $3(10.7)$ & 0.110 \\
\hline $\begin{array}{l}\text { Piperacillin/tazobactam, } \\
\text { No. (\%) }\end{array}$ & $15(27.3)$ & $10(37.0)$ & $5(17.9)$ & 0.694 \\
\hline $\begin{array}{l}\text { Cefoperazone/sulbactam, } \\
\text { No. (\%) }\end{array}$ & $30(54.5)$ & $14(51.9)$ & $16(57.1)$ & 0.266 \\
\hline $\begin{array}{l}\text { bAnti-fungles, No. (\%) } \\
\text { 45(81.8) }\end{array}$ & $20(74.1)$ & $25(89.3)$ & \\
\hline $\begin{array}{l}\text { aAnti-MRSAs included glycopeptides, linezolid, and daptomycin. } \\
\text { bAnti-fungles included fluconazole, voriconazole, posaconazole, caspofungin and micafungin. }\end{array}$ & \\
\hline
\end{tabular}

Table 3. Univariate and multivariate analysis of risk factors associated with hemorrhagic pneumonia in hematological patients with SM bacteremia 


\begin{tabular}{|c|c|c|c|c|}
\hline \multirow[t]{2}{*}{ Characteristics } & \multicolumn{2}{|l|}{ Univariate analysis } & \multicolumn{2}{|l|}{ Multivariate analysis } \\
\hline & $\begin{array}{l}\text { Hazard ratio }(95 \% \\
\mathrm{Cl})\end{array}$ & $\begin{array}{l}P- \\
\text { value }\end{array}$ & $\begin{array}{l}\text { Hazard ratio }(95 \% \\
\mathrm{Cl})\end{array}$ & $\begin{array}{l}P \text { - } \\
\text { value }\end{array}$ \\
\hline Age & $\begin{array}{l}1.030(0.994- \\
1.068)\end{array}$ & 0.105 & & \\
\hline Polymicrobial bacteremia & $\begin{array}{l}1.917 \rrbracket 0.490- \\
7.494 \rrbracket\end{array}$ & 0.350 & & \\
\hline Neutropenia & $\begin{array}{l}5.176(1.252- \\
21.411)\end{array}$ & 0.023 & $\begin{array}{l}4.965(1.010- \\
24.403)\end{array}$ & 0.049 \\
\hline Hemoglobin $<60 \mathrm{~g} / \mathrm{L}$ & $\begin{array}{l}1.261(0.433- \\
3.668)\end{array}$ & 0.671 & & \\
\hline Platelet $<50,000 / u L$ & $\begin{array}{l}1.500(0.230- \\
9.763)\end{array}$ & 0.671 & & \\
\hline Fibrinogen $<2 g / L$ & $\begin{array}{l}2.100(0.537- \\
8.217)\end{array}$ & 0.286 & & \\
\hline PT $>13.5 s$ & $\begin{array}{l}2.692(0.883- \\
8.206)\end{array}$ & 0.082 & $1.022(0.255-4.101)$ & 0.975 \\
\hline APTT $>33.5 \mathrm{~s}$ & $\begin{array}{l}2.265(0.694- \\
7.389)\end{array}$ & 0.175 & & \\
\hline D-Dimer>700ug/L FEU & $\begin{array}{l}1.568(0.389- \\
6.319)\end{array}$ & 0.527 & & \\
\hline Albumin $<30 \mathrm{~g} / \mathrm{L}$ & $\begin{array}{l}1.440(0.487- \\
4.255)\end{array}$ & 0.509 & & \\
\hline Globulin<20g/L & $\begin{array}{l}1.261(0.433- \\
3.668)\end{array}$ & 0.671 & & \\
\hline C-reactive protein $\geq 10 \mathrm{mg} / \mathrm{dl}$ & $\begin{array}{l}1.973(0.638- \\
6.106)\end{array}$ & 0.238 & & \\
\hline Procalcitonin $>0.5 \mathrm{ug} / \mathrm{L}$ & $\begin{array}{l}9.375(1.849- \\
47.522)\end{array}$ & 0.007 & $\begin{array}{l}11.241(1.825- \\
69.241)\end{array}$ & 0.009 \\
\hline HSCT & $\begin{array}{l}1.167(0.335- \\
4.060)\end{array}$ & 0.809 & & \\
\hline $\begin{array}{l}\text { Inadequate initial antimicrobial } \\
\text { treatment }\end{array}$ & $\begin{array}{l}1.451(0.481- \\
4.377)\end{array}$ & 0.508 & & \\
\hline Central venous catheter & $\begin{array}{l}2.717(0.479- \\
15.402)\end{array}$ & 0.259 & & \\
\hline $\begin{array}{l}\text { Prior tigecycline therapy within } 1 \\
\text { month }\end{array}$ & $\begin{array}{l}4.364(1.383- \\
13.772)\end{array}$ & 0.012 & $\begin{array}{l}4.465(1.108- \\
18.000)\end{array}$ & 0.035 \\
\hline $\begin{array}{l}\text { Prior carbapenem therapy within } 1 \\
\text { month }\end{array}$ & $\begin{array}{l}1.250(0.297- \\
5.256)\end{array}$ & 0.761 & & \\
\hline
\end{tabular}




\section{Figures}

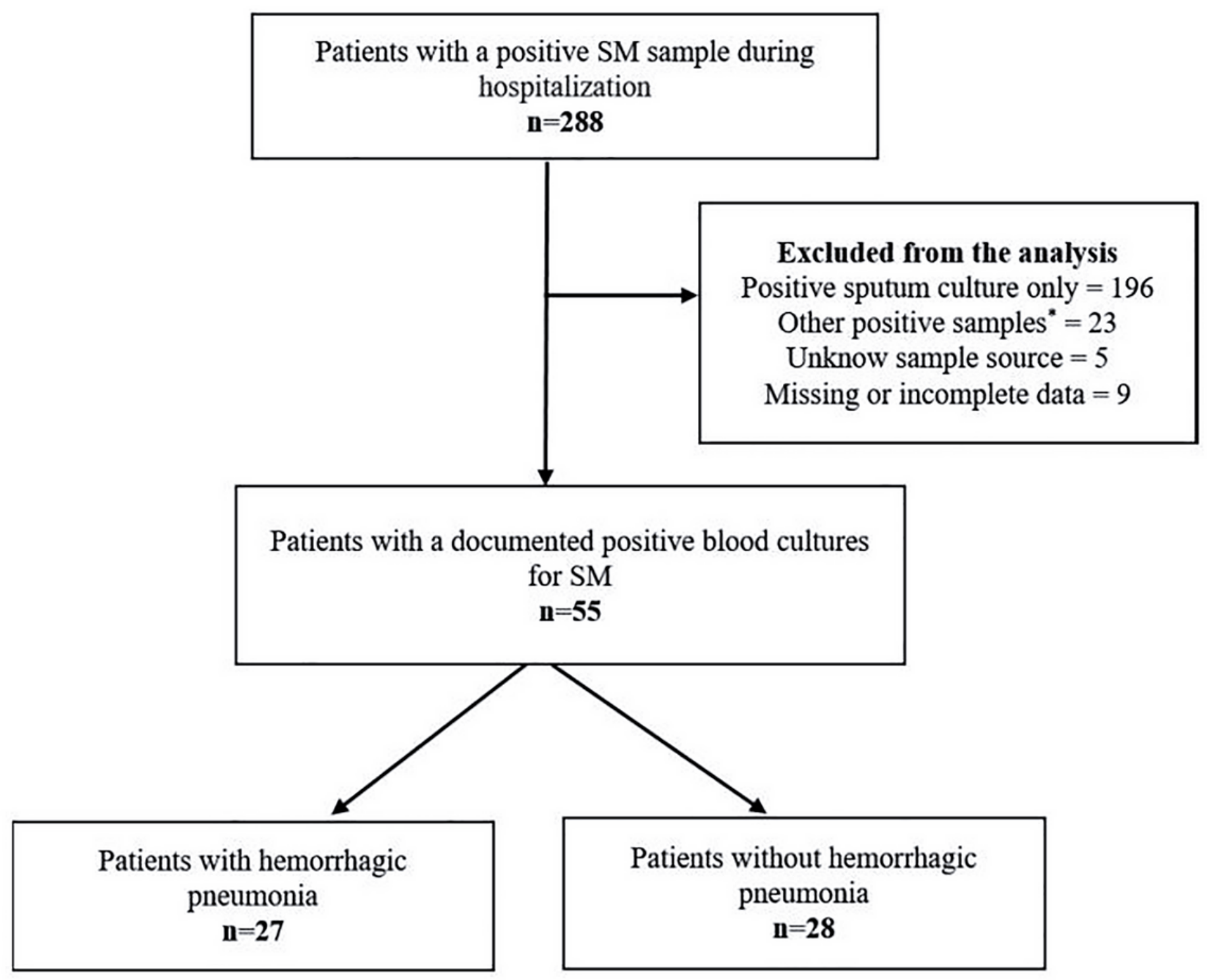

Figure 1 
Flowchart of the inclusion of patients presenting with SM bacteremia in hematological patients. Asterisk means that culture samples from skin or wound $(n=9)$, pharyngeal swab $(n=6)$, stool $(n=5)$ and urine $(n=3)$.
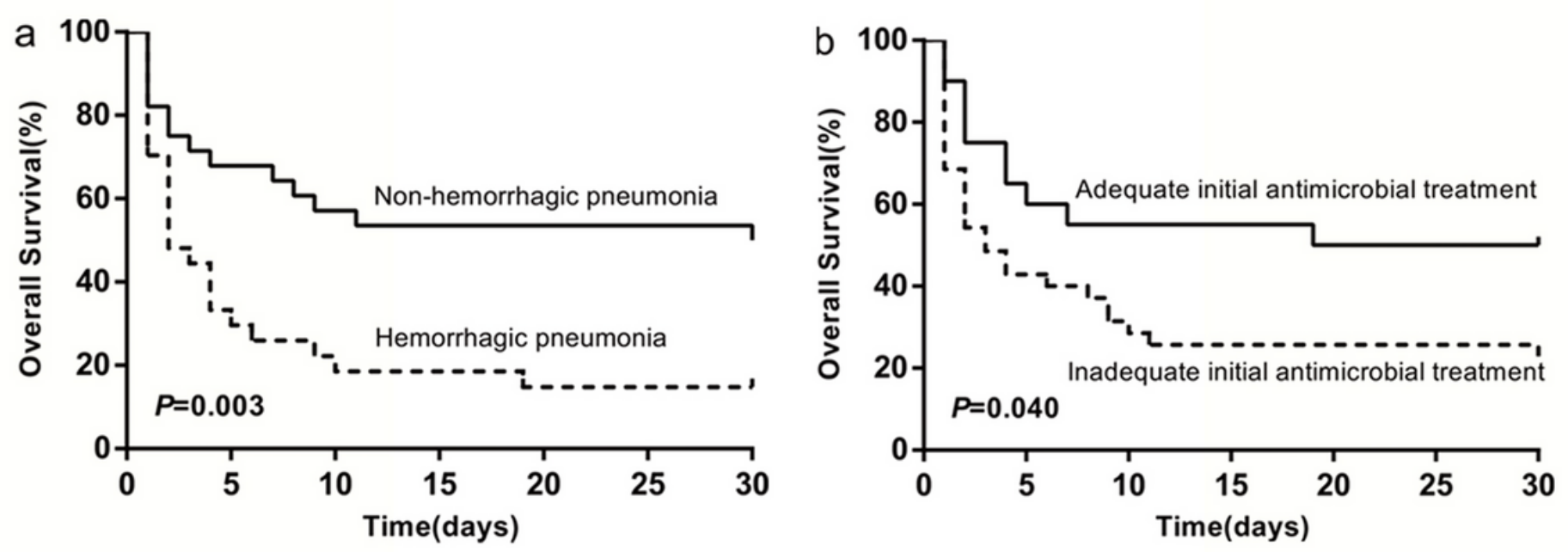

Figure 2

Kaplan-Meier curves of 30-day overall survival (OS) in patients with SM bacteremia. a OS was significantly lower in patients with hemorrhagic pneumonia. b OS was significantly lower in patients with inadequate initial antimicrobial treatment.

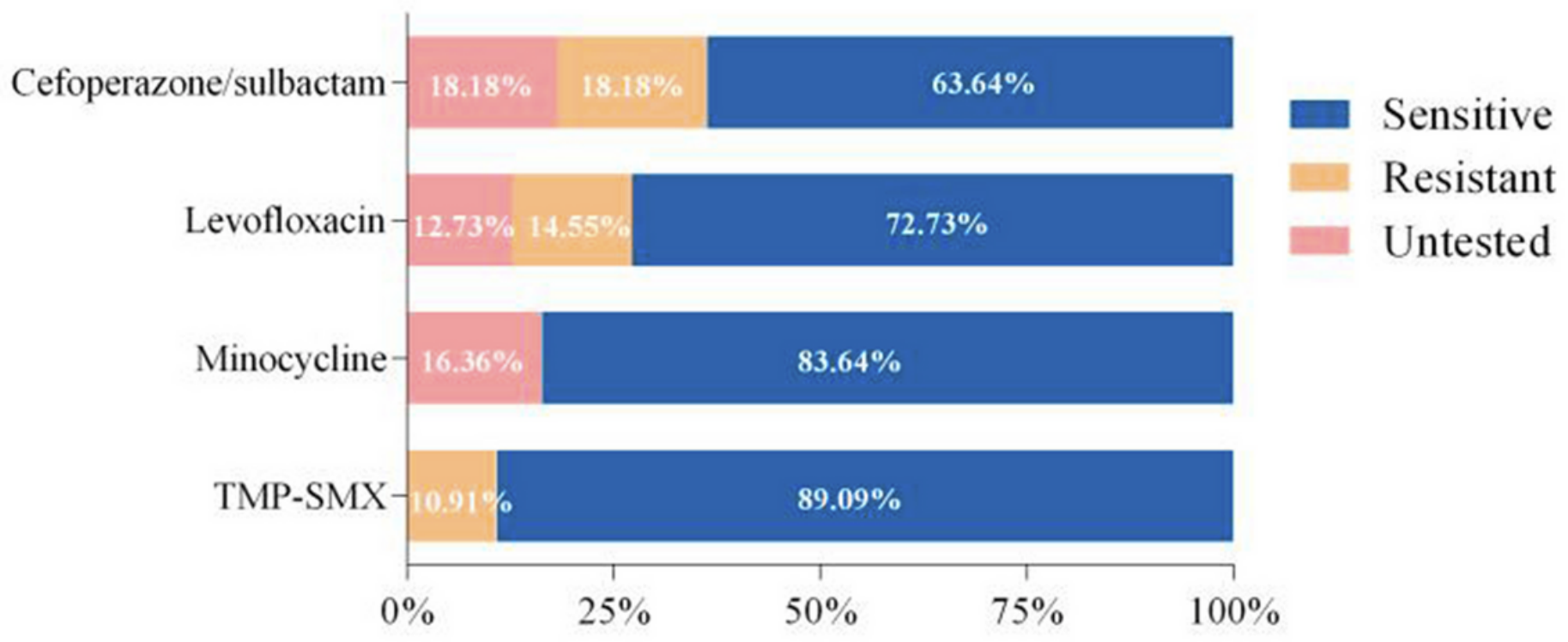

\section{Figure 3}

In vitro antimicrobial susceptibility for SM isolates 